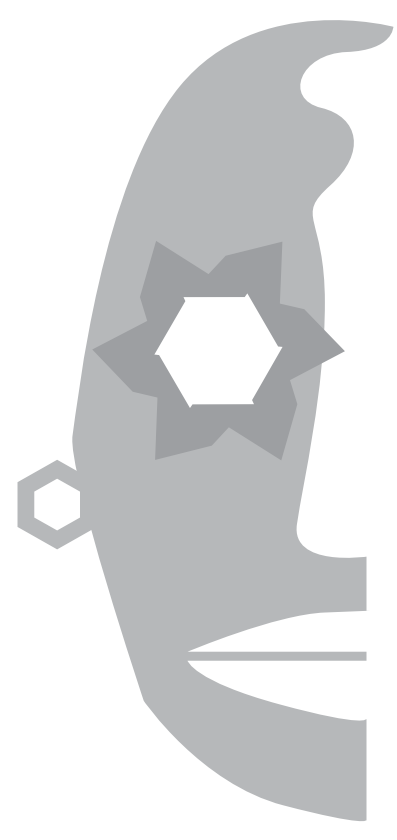

Cuauhtémoc Pérez

Medrano

Universidad de Potsdam

\section{Insularidad y meteorología: espacios re-expresados y sub-versivos en la narrativa contemporánea cubana}

\section{Resumen}

Cuba es un país insular en donde los fenómenos meteorológicos han sido elementos metafóricos importantes en la configuración de los mitos fundacionales. Será útil entonces observar los fenómenos histórico-socioculturales como fenómenos meteorológicos, pues matizarían cuestiones locales y globales, en lo referente a la identidad y diversidad cultural, estudios estéticos y literarios.

La insularidad de Cuba ha pasado por distintos estadios de análisis, desde el plano ontológico hasta el plano político o literario. La interacción entre espacio imaginado y expresado en la literatura exige un acercamiento mediado entre los referentes "reales" y meta-literarios. La utilización de la metáfora de la isla ha sido perenne en la historia cultural cubana. Las generaciones de escritores en Cuba, desde hace treinta años aproximadamente, buscan superar algunas barreras ideotemáticas y estilísticas mediante la re-expresión de sus propias sub-versiones literarias. Para ellos, la insularidad o la isla se convierten en un tópico versátil que tensa las relaciones entre las estéticas y sus categorías. Tales fenómenos literarios serían perceptibles en la narrativa de Abilio Estévez, Marilyn Bobes y Antonio José Ponte, lo cual presenta un debate interdisciplinario centrado particularmente en el posicionamiento global de la narrativa cubana y las contemporáneas subversiones literarias.

Palabras clave: Cuba, isla, meteorología, literatura 


\begin{abstract}
On the island of Cuba, meteorological phenomena have been important metaphorical elements in the configuration of the country's creation stories. For this reason, it could be useful to observe historical, sociocultural phenomena as meteorological in nature as that would explain many local and global questions regarding cultural identity and diversity, and aesthetic and literary studies.

Insularity in Cuba has been analyzed from several different perspectives ranging from ontological to political or literary in nature. The interaction between real and imaginary space in literature demands an approach somewhere between "real" and meta-literary. Throughout the cultural history of Cuba, use of the island metaphor has been a constant and for the past 30 years or so, generations of Cuban writers have sought to overcome idiothematic and stylistic obstacles through the re-expression of their own literary subversions. For these authors, insularity or the island itself becomes a versatile topic that places stress on the relationship between aesthetical aspects and their categories. These literary phenomena would be noticeable in the stories of Abilio Estevez, Marilyn Bobes and Antonio Jose Ponte, which would give rise to an interdisciplinary debate surrounding the global positioning of Cuban fiction and contemporary literary subversions.
\end{abstract}

Keywords: Cuba, island, meteorology, literature

\title{
Introducción
}

Históricamente, para la literatura cubana la insularidad o la imagen de la isla ha sido no sólo una referencia geográfica, sino también un punto que condensa las posibilidades referenciales y las vivencias culturales dentro del espacio insular (Mateo Palmer, Álvarez, 2005: 89). En otras palabras, la insularidad representa para Cuba un cúmulo de valores sociales y culturales que se manifiestan en sus literaturas nacionales a través de la representación del espacio. La isla se encontraría geográficamente a la intemperie y a merced de los fenómenos atmosféricos que involucran su representación ${ }^{1}$. Por esa razón, en este trabajo los términos insularidad y meteorología se vinculan para reflejar, primero, una correlación en la representación del espacio en la narrativa; y por otro lado, los fenómenos socio históricos o culturales bajo la alegoría de "meteoro", ya que modifican o evidencian los matices de las re-expresiones y las sub-versiones de la cualidad insular en la narrativa cubana. El uso alegórico de la meteorología no es gratuito, ya que en muchos casos los vientos, los ciclones y fenómenos atmosféricos representan un factor muy importante en la configuración de la insularidad. Alegóricamente los meteoros, asumidos como sociohistóricos, poseen una relación muy importante en la expresión de la vivencia cultural insular.

Desde hace treinta años, aproximadamente, generaciones de narradores en Cuba oscilan entre los vientos desorientadores de la ruptura, adecuación y rearticulación

1 Según el Diccionario de la Real Academia de la Lengua, meteorología (del gr. $\mu \varepsilon \tau \varepsilon \omega \rho o \lambda$ oүía) es la "ciencia que trata de la atmósfera y de los meteoros" (RAE, 2015). Según el mismo diccionario, "meteoro" sería entonces algún "fenómeno atmosférico, que puede ser aéreo, como los vientos, acuoso, como la lluvia [...], luminoso, como el arco iris, el parhelio o la paraselene, y eléctrico, como el rayo [...]" (RAE, 2015). 
de algunas barreras ideo-temáticas y entre el viento nordeste de las estilísticas en el ejercicio literario que emanan de nuevas maneras de observar su mundo. En sus obras quedan expresados y re-expresados la sensibilidad y la experiencia de sus propios procesos y fenómenos socioculturales. Podría decirse que las versiones de la isla se han diferenciado, creando posturas y símbolos sub-versivos a lo largo de la historia de la literatura considerada como nacional en Cuba. Lo que sigue a continuación es un análisis sobre los modos de representar la isla y las correlaciones con la noción de insularidad frente a sus procesos sociohistóricos en las obras de los narradores finiseculares: Abilio Estévez, Marilyn Bobesy y Antonio José Ponte. Nos preguntamos sobre las relaciones histórico-culturales y la narrativa cubana en las últimas décadas para vincular el ejercicio literario, cultural y estético.

\section{Meteoros socio-históricos}

Desde los años setenta se comenzaron a ennegrecer algunos nubarrones en la ideología revolucionaria cubana; más tarde los primeros rayos de los chubascos terminaron por hacer visibles las grietas y los baches de los procesos revolucionarios. En las siguientes décadas, con la tormenta del masivo de exilio cubano y con la borrasca del, así llamado Caso Ochoa, se demarcó la eventual agreste ruptura del bloque soviético y la subsecuente tormenta del Periodo Especial Tiempos de $\mathrm{Paz}^{2}$ de los años noventa, caracterizada por el desabasto de insumos energéticos y de industria, desempleo, pauperización de la sociedad y la migración. El envío de remesas, desde Miami principalmente, y el turismo se consolidarán como los medios más redituables para mitigar la crisis. Quienes se quedaron en la isla tuvieron que encontrar la manera para sobrevivir con una moneda local insuficiente y desequilibrada y el ejercicio de algunas prácticas ilegales. Las idealizaciones de Cuba como país de vanguardia y revolucionario se desmoronaron; los valores cohesionadores del "hombre nuevo", planteados por Ernesto Guevara, se olvidaban cuando las necesidades más primarias se volvían inabarcables. El discurso oficial buscaba de alguna manera continuar con un desgastado ideal utópico revolucionario mientras la carencia y el aumento de algunos problemas sociales diferían de estas idealizaciones (Fornet, 2003: 3-7).

\section{Meteoros estéticos-culturales}

De entre los agrietados contextos sociales, económicos y políticos de los años noventa, generaciones de escritores cubanos fungieron desde la literatura y, en muchos casos, como el ojo periodista o cronista de una ruda realidad que distaba mucho del ideario utópico revolucionario cubano. Quienes se dedicaron a narrar los espacios de la isla lo hicieron por medio de personajes que, aunque tangibles

2 En términos generales, entre 1991 a 1997 en Cuba, se llevaron acabo medidas económicas y políticas que se realizaron y que poseen una estrecha relación con la caída del Muro de Berlín bajo el contexto global. 
en la realidad, eran inéditos en las letras cubanas. El morbo por percibir las realidades devastadas por la Guerra fría después del Muro de Berlín, se volvió para muchas editoriales extranjeras no sólo una exquisitez, sino una novedad de venta. En este sentido, Cuba no fue excluida de ese morbo comercial, y la literatura cubana se caracterizó por la exacerbación de personajes apócrifos dentro y fuera del espacio literario insular cubano, personajes, ambientes y temas que poco a poco se convirtieron en lugar común (Strausfeld, 2000: 11-23). En este contexto, tanto la insularidad o la representación de la isla como sus connotaciones, símbolos y referencias, mostraron nuevas versiones, o quizá mejor dicho sub-versiones de las sobrevivencias culturales. La crítica local empleó términos útiles para poder reformularse a la sombra de algunos emblemas "revolucionarios", de ahí que la posmodernidad o el calificativo postsoviético se volvieran categorías rentables, quizá solo para demostrar que, aunque retraída, la isla mantenía aún un paso propio y similar al de las letras latinoamericanas, caribeñas y globales, es decir, "el sueño de la vanguardia por la vanguardia, las artes y las letras [que tienden a] asumir todos lo patrimonios posibles y de tratar de modificarlos mediante la violación de los códigos patrimoniales" (Vázquez Montalbán,1996: 12).

La lectura o reinserción de autores antes silenciados en el exilio o en el insilio, provocó que las generaciones de escritores de los años noventa se colocaran en una extraña frontera de tolerancia en donde se han enfrentado y reconocido frente a los diversos posicionamientos sobre la literatura y la crítica cubanas (Rojas, 2009: 192-207). El espacio insular expresó puntos de confluencia, no menos conflictivos con el imaginario social revolucionario, que se enfrentaban con las necesidades individuales. Dichos conflictos serían perceptibles en la narración y poetización de los espacios antes plurales y ahora individualizados y representados en la ficción. En el inicio de la primera década del siglo XXI, la dislocación de contextos y símbolos se había reforzado y se hizo patente la diversidad del narrar la isla, es decir, la isla como imagen o símbolo se complejizó en cuanto a las referencias simbólicas de su espacio mismo.

\section{Meteorología e insularidad}

Salvador Redonet-Cook, en la publicación de la antología Los últimos serán los primeros, (Redonet, 1993) buscó compaginar una generación de escritores dispares que respondían ideotemática y particularmente a su realidad. Más tarde con el término que los agrupó como novísimos se hacía tangible la posibilidad de unir la crítica con la praxis literaria plural. Haciendo uso de los recursos más variados, los novísimos reforzaron, como señala Ana Araujo:

En lo ideotemático [...] por la alteración de ciertas estructuras formales: la reiteración del texto minimalista, la superposición de planos -realidad/irrealidad, 
lógica/absurdo, vigilia/sueño-, la transgresión de la linealidad y la fragmentación discursiva en el relato, los juegos intertextuales -incluso con un texto propio-, funcionan para unas nuevas semántica y pragmática. (Araujo, 1998-9: 213).

Pero no sólo eso, sino que también en la misma reorganización de los modelos narrativos se incluyen algunas tendencias que buscan poner entredicho el concepto de realidad:

Están [...] las sombras del neobarroco y el absurdo, los estilos asimétricos de Severo Sarduy, Virgilio Piñera y Calvert Casey, en esa agónica lucha por el eliminar del relato la estructura dramática iniciada por el cuento realista. Sus ficciones son hijas del minimalismo y la posmodernidad, y algunos de sus textos resultan un verdadero rompecabezas para la crítica. Son historias audaces, desprovistas de centro, cuyo tono se acerca al ensayo, la poesía y la literatura de reflexión (López Sacha, 2006: 252).

Esas tendencias se desenvolvieron con más ímpetu en las generaciones de escritores posteriores al final del milenio pasado, como afirma Salvador Redonet (Redonet, 1999: 8-9). Impulsados por la innovación como fin último del quehacer literario, los narradores buscarían la desmitificación de mitos nacionales a través de la ironía o la sátira, la acentuación del elipsis o la analogía, la ambigüedad, el sentido apocalíptico, caótico, fragmentario, lúdico o en montaje de estructuras sumergidas bajo un predominio de lo discursivo en cuanto a los modos de narrar la historia (Redonet, 1999: 16), entre otros recursos y estrategias. La representación de la isla estaría relacionada con una actitud desmitificadora, pero también con los ambientes caótico o apocalíptico, mientras que la fragmentación sería estilística, simbólica y discursiva, atenta a la readecuación de discursos de una insularidad negativa. La isla, al ser un espacio delimitado, puede ser relacionada con la reclusión o el anatema de la incomunicación o la locura. Es en este sentido que se percibe cierta reactivación del sentimiento de negatividad insular en la propagación del planteamiento del verso escrito por Virgilio Piñera en 1943: "[L] a maldita circunstancia del agua por todas partes" (Piñera, 2011: 29) y de autores que, más contemporáneamente, refrendan esa tradición del no, como Antón Arrufat (Ponte 1993: 10-15). La locura sufrida dentro y fuera del aislamiento ha encontrado ejemplos ignorados en la tradición literaria reciente; una muestra sería el narrador Guillermo Rosales, en El juego de la viola (1967), o el caótico y lúdico Boarding home $(1987)^{3}$, y quien se suicidó en el exilio en 1993.

3 Son pocos los textos que han recuperado la obra de Guillermo Rosales. Apenas en estos últimos años han comenzado a surgir algunos trabajos. Véase el recién trabajo de Elizabeth Mirabal y Carlos Velazco (2013). Hablar de Guillermo Rosales, Miami: Editorial Silueta. 
El viaje y la relación entre la tierra y el mar han generado en la isla la concepción de un doble límite que ubica el análisis de su propia meteorología. La tierra no es más segura que la inestabilidad del mar; por eso mismo, en la metáfora de la isla se observa un doble viaje, uno hacia la inestabilidad de la historia misma y, por otro lado, uno hacia la irremediable delimitación a ese mismo desequilibrio que, para el escritor cubano en general, ha representado la isla. La tierra "firme", como ha sugerido Reinaldo Arenas en El color del verano (1991), o Iván de la Nuez, es una balsa perpetua, un frágil trozo de tierra que se tambalea en el mar (Nuez, 1998), frente a los meteoros sociohistóricos que la zarandean.

Por ese motivo, la insularidad sólo puede ser expresada por espacios plurales que prolongan la diversidad de versiones y sub-versiones de sí misma. Así, La isla que se repite (1998), de Antonio Benítez Rojo, es una manera de configurar las trayectorias de las representaciones de la isla en los espacios culturales insulares. Lo es también la idea de fractalización de la isla y sus capacidades de relación en una mise en abyme, como sugiere Ottmar Ette (Ette, 2005: 123-155), ya que representa la vinculación de los distintos espacios que pueden ser perceptibles en la literatura como ejercicio y conocimiento de vida (Ette, 2004: 11-24). La imagen de la isla no es un reflejo de la realidad, sino que surca el plano de la representación y se consolida como un capital simbólico que entrelaza valores ideológicos y sociales con la obra artística (Par Kumaraswami, Kapcia, 2010: 184-185). En esta praxis se han conjugado tangentes del estado teleológico y el estado negativo del ser "cubano", es decir, "valores, - o disvalores- [...que...] cuestionan la idea -o el mito- de un alma, una civilización, un espíritu, una cultura y hasta de una nacionalidad cubana" (Rojas, 1998: 117). Estas ideas problematizan a la isla como capital simbólico sociocultural y estético, y sus tránsitos muestran una serie de rasgos, caras, facetas relacionados con los efectos de los meteoros históricos y socioculturales que se hacen evidentes en los modos de re-expresar la insularidad y las sub-versiones de esta. A continuación, se ofrece el ejemplo de tres narraciones en las que se evidencia la presencia de los efectos meteorológicos históricos, socioculturales y estéticos en la narrativa cubana a través de la imagen de la isla.

\section{La narrativa cubana contemporánea}

Abilio Estévez publicó en España la novela Tuyo es el reino (1997). En ella, Estévez construye una isla como tópico a través de toda la mitología alrededor del espacio insular. Un año más tarde, en El horizonte y otros regresos (1998), recuperó algunas fabulas y narraciones que fueron escritas casi al mismo tiempo que la novela, por lo que mantienen una comunicación muy estrecha con el estilo y con ciertas temáticas trabajadas en Tuyo es el reino. En el apartado Tres leyendas autobiográficas dentro del libro de cuentos, se encuentra la Historia de Hans de Islandia y Arodi la Cantante. La narración fabula cuasi mitológicamente el origen 
de ciertas características poco comunes en el paisaje de la isla de Cuba, como un jardín exótico. Estévez centra la historia en la relación amorosa e idealizada entre Hans y Arodi. Él es un personaje extranjero en Cuba, y al no saberse su procedencia exacta en el relato, el narrador la asigna el mote de Hans de Islandia. El nombre del personaje podría referir, por un lado, a la primera novela casi homónima de Víctor Hugo, Han d'Islande (2010 [1836]), donde Han es un personaje que se aisla del mundo con un oso; pero por otro lado, y lo más importante, es que al ubicar al personaje procedente de Islandia, el narrador recupera el empalme de dos distintas insularidades, la que se "vive" en la ficción al ubicar el cuento en Cuba y la del pasado ficcionalizado del personaje, procedente de otra Isla.

Arodi, la Cantante, es la contraparte amorosa de Hans. La pareja es una versión idealizada de la pareja platónica, un juego de espejos romántico de una pareja que se complementa. A la muerte de ella se desarrollan algunos cambios en la conducta de Hans: se vuelve taciturno, callado, y construye un jardín en donde se pueden encontrar arboles exóticos como homenaje a la memoria de Arodi, y es como comienza la narración in media res:

Aquí hay ahora un jardín con álamos, sauces, cipreses, olivos y hasta un esplendido sándalo rojo de Ceilán. Yo sé que es difícil creerlo. En esta isla de árboles anodinos, uniformes, con el mismo verdeaburrido, no se conciben sauces, ni cipreses, aunque lo escriban cien incansables elegiacos como el desdichado autor de Fidelia o la necrófaga poetisa de La vuelta al bosque. (Estévez, 1998: 97)

En términos generales, el cuento insinúa una insularidad mítica, en vínculo con la vegetación insular. La descripción del paisaje fuera del jardín se puede interpretar como un hartazgo o negatividad frente de la homogeneidad del paisaje "revolucionario". No es gratuito que los adjetivos para describir los árboles de la isla sean "anodinos", "uniformes" y "verdeaburridos", para resaltar la singularidad del jardín forjado por el mítico Hans, pero también para sugerir el cansancio de vivir bajo los parámetros de igualdad revolucionaria. En el fragmento citado, aparecen dos títulos de poemas de Juan Clemente Zenea y Luisa Pérez de Zambrana, respectivamente, escritores de la Cuba decimonónica y referentes de la historia literaria nacional cubana. En resumen, se desarrolla la idea de una insularidad delimitada y construida como un espacio utópico a través de la mitificación de personajes apócrifos y por tanto ficcionalizados. Los meteoros histórico-sociales se perciben en un sentimiento de negatividad frente a un aislamiento "uniforme" y "verdeaburido", y en la necesidad de "nuevos" mitos culturales y literarios.

Otra mirada de una sub-versión de la imagen de la isla se halla en la interiorización de los efectos sociohistóricos en el marco del periodo especial, como sucede con el el cuento Pregúntaselo a Dios, de Marilyn Bobes, que forma parte del libro de relatos Alguien tiene que llorar (1995). A manera de montaje fílmico, en el 
cuento se relata la historia epistolar de una cubana que se casa con un francés y deja Cuba. Así sabemos que la cubana lleva el nombre de Iluminada Peña y que conoció a Jaques Dupuis, su esposo, en la Cuba del periodo especial. Los temas que se abordan en un tono apocalíptico y nostálgico son la prostitución, los apagones y la carencia habanera.

De las cartas escritas por el personaje, Iluminada Peña, conocemos el choque cultural que representó su nueva vida en Toulouse. Resaltemos a partir de aquí algunos aspectos que matizarían la sub-versión de la imagen de la isla; primeramente, el nombre mismo del personaje, "Iluminada Peña", sugiere una piedra grande, un cerro con luz, que por oposición simbólica contrasta con el ambiente oscuro de los constantes apagones del periodo especial. El nombre del personaje podría percibirse como una sinécdoque de la isla al visualizar en él una piedra grande que, después de partir al extranjero, queda flotando entre sus recuerdos y el espacio ajeno. El personaje femenino genera un efecto de simultaneidad entre el espacio insular y su propio cuerpo e historia; por ello externa ciertas complejidades del aislamiento y el extrañamiento que se vinculan a las perspectivas y las maneras en las cuales el personaje describe fragmentos del recuerdo de la isla: "Se sentaron a una mesa desde la que se divisaba un gran tramo de la ciudad. Él improvisó un mapa sobre una servilleta y le indicó el lugar exacto donde se encontraban. Entonces Iluminada comprendió por qué veía el Malecón como si estuviera en un barco: del mar hacia la tierra y no del muro hacia el mar (Bobes, 1995: 65).

Debe subrayarse que en este fragmento se narra la primera vez que hay un cambio de perspectiva entre la isla, en tanto espacio geográfico, y donde la tierra firme parece ajena a la perspectiva del personaje, por lo que la perspectiva sugiere un paradójico lazo de extrañamiento o ajenidad del lugar propio. Este detalle se repetirá y acrecentará al distanciarse de su espacio natal, pues Iluminada regresa de vacaciones a Cuba después de una estancia en Toulouse, y mira su isla: "Frente a ella se extiende el mar oscuro. Iluminada lo mira en silencio, desde la ciudad también oscura."(Bobes, 1995: 69). Con la oscuridad, la ciudad y el mar se han vuelto un reflejo uno de otro, la tierra fija no existe cuando el personaje ha perdido pertenencia. El personaje dictamina: "Me voy mañana, anuncia con voz neutra, y su amiga se cuelga de su cuello e Iluminada siente su olor a perfume barato y sufre también por ese olor que ya no le pertenece" (Bobes, 1995: 69). La simultaneidad entre espacio y cuerpo se rompe al final de la narración; el ambiente nostálgico generado en las narraciones epistolares se opone a una realidad de ajenidad del espacio pero, paradójicamente, conserva la sensación de perdida o de reformulación de la propia historia, justo en los años acres después de 1989.

Otro tipo de sub-versión insular es el que se presenta en el libro de relatos Un arte de hacer ruinas y otros cuentos, de Antonio José Ponte (2005), en especial el cuento 
Un arte de hacer ruinas. La historia narra la inquietud de un joven urbanista que quiere desarrollar una tesis sobre las "barbacoas"4 basándose en el Tratado breve de la estática milagrosa. Se genera un misterio alrededor de la existencia de algunos seres llamados "tugures". El personaje central y el asesor de la tesis descubren, entre tonos detectivescos y de ciencia ficción, que existe un mundo doble en ruinas debajo de La Habana. Tras la muerte en un derrumbe del "Profesor D", autor del Tratado breve.... se devela la existencia de Tuguria, "la ciudad hundida, en donde todo se conserva como en la memoria" (Ponte, 2005: 73), una ciudad imaginaria e idealizada construida con los trozos que se derrumban en la realidad.

El espacio habanero se describe arruinado, como metonimia de la isla en los años noventa, y los efectos de devastación económica son visibles en la metaforización de los seres y del espacio. El paisaje de la isla tiende hacia la interiorización y la segmentación del espacio un plano vertical. Como las barbacoas, la isla no es solo horizontal sino que se adecua a la verticalidad del espacio, como afirma el narrador del cuento: "Cuando no encuentras tierra nueva, cuando estás cercado, puede quedarte todavía un recurso: sacar a relucir la que está debajo de lo construido. Excavar, caminar en lo vertical. Buscar la conexión de la isla con el continente, la clave del horizonte". (Ponte, 2005: 66-67).

El espacio insular hace suya la posibilidad de ser vertical para luchar contra el encierro; la isla se perfora y se multiplica hacia el interior, cada espacio vertical forma parte de esa horizontalidad que permite conectarse con la continentalidad. En otras palabras, la restructuración de ese espacio sugiere un viaje hacia el interior, no hacia el origen sino hacia otros mundos o sub-versiones de esos espacios, de esas islas que se mantienen en la memoria.

\section{A modo de conclusión}

La utilización simbólica de la meteorología hace manifiestos los efectos de los meteoros sociohistóricos y estéticos culturales en la conformación simbólica de la isla. Aunque son dos rubros que podrían ser divisibles, se ha intentado no hacer tan tajante segmentación, pues se asume que están íntimamente relacionados como fenómenos meteorológicos y son parte de un modo de observar y vivir el mundo en este período de la literatura cubana.

La representación de huracanes, ciclones y demás meteoros marítimos se han dejado fuera del estudio para no pecar de obviedad en el análisis del espacio insular, ya que es un tópico que requeriría un estudio aparte en la literatura cubana. Se ha buscado ante todo tratar de hacer visibles esas simbologías de la isla que

4 Las barbacoas son construcciones domésticas en forma de literas, muy comunes en Cuba para encarar el aumento poblacional en La Habana y el desabasto de habitación. 
se hallan subyacentes a otras temáticas y que se manifiestan como sub-versiones. En cuanto a los ejemplos presentados, se percibe una dinámica simbólica en las descripciones del espacio. Si bien el espacio ficcional presentado por Estévez en su cuento se centra en la dualidad entre la ambigüedad de la realidad y ofrece una mitología de la conformación de un jardín exótico en la isla, también es cierto que está construido desde la simulación del discurso autobiográfico. Este hecho se encuentra en correspondencia discursiva con los otros dos cuentos.

Para el caso del cuento de Bobes, la narración está conformada por la fragmentación construida por el simulacro epistolar, modo como se vincula a la narración que toca los planos autobiográficos. Los sucesos narrados parten de la memoria del personaje Iluminada Peña, para construir la propia memoria que le hace confrontarse con su presente desarraigado y que simula una empatía y empalme con la autora. Distinto es el proceso que sufren los personajes descritos en el cuento de Antonio José Ponte ya que, aunque también están forjados bajo el discurso autobiográfico, los hechos se asumen bajo la ambigüedad realidad/ ficción. El rasgo en el que coinciden tanto Ponte como Bobes está en la sensación de alejamiento, no físico o territorial pero sí temporal, lo que crea una lectura que refiere la construcción de la memoria. En este sentido, los tres ejemplos son sincrónicos, ya que presentan un interés en la reacción de la memoria, sea lejana o de un pasado cercano; sea en la reactivación de mitos de la historia literaria cubana (en el caso de Estévez), sea en la re-expresion de una insularidad vivencial (en el caso de Bobes), o bien se trate de una tradición negativa de la insularidad (en el caso de Ponte). En suma, cada uno de los cuentos se presenta como un espacio re-expresado o una sub-versión de la isla en la narrativa cubana contemporánea.

\section{Referencias}

Araujo, Ana (1998-1999). El espacio otro en la escritura de las (novísimas) narradoras cubanas. Temas, 16/17, 212-217.

Arenas, Reinaldo (1991). El color del verano: Miami, Fla.: Ed. Univ.

Benítez Rojo, Antonio (1998). La isla que se repite, Barcelona: Ed. Casiopea.

Bobes, Marilyn (1995). Alguien tiene que llorar, La Habana: Casa de las Américas.

Estévez, Abilio (1998). El horizonte y otros regresos, Barcelona: Tusquets. . (1997).Tuyo es el reino, Barcelona: Tusquets.

Ette, Ottmar (2005). ZwischenWeltenSchreiben: Literaturenohnefesten Wohnsitz, Berlin: Kulturverl. Kadmos.

. (2004). Die Aufgabe der Philologie, Berlin: Kulturverl. Kadmos. 
Fornet, Jorge (2003). "La narrativa cubana entre la utopía y el desencanto", Hispamérica, 32 (95), 3-20.

Hugo, Victor (2010). Han de Islandia, Buenos Aires: Losada.

López Sacha, Francisco (2006). Pastel flameante, La Habana: Ed. Letras Cubanas.

Mateo Palmer, Margarita, Álvarez Álvarez, Luis (2005). El Caribe en su discurso literario, Santiago de Cuba: Ed. Oriente.

Nuez, Iván, de la (1998). La balsa perpetua: soledad y conexiones de la cultura cubana, Barcelona: Ed. Casiopea.

Par Kumaraswami, Antoni Kapcia (2010). Hacia un entendimiento mejor de la cultura literaria en la Cuba revolucionaria. Araceli Tinajero (Edit.) Cultura y letras cubanas en el siglo XXI, Madrid: Iberoamericana, 177-191.

Piñera, Virgilio (2011). La isla en peso, La Habana: Ediciones Unión.

Ponte Antonio José (2005). Un arte de hacer ruinas y otros cuentos, México, D.F: Fondo de Cultura Económica.

. (1993). La lengua de Virgilio, Matanzas, Cuba: Ed. Vigía.

Redonet, Salvador (prolg.) (1999). Para el siglo que viene: (post)novísimos narradores cubanos, Zaragoza: Prensas Universitarias de Zaragoza.

. (1993). Los últimos serán los primeros, La Habana: Editorial Letras Cubanas.

Rojas, Rafael (1998). Isla sin fin: contribución a la crítica del nacionalismo cubano. Miami, Fla.: Ed. Universal.

. (2009). El estante vacio: literatura y politica en Cuba. Barcelona: Anagrama.

Rosales, Guillermo (2004). Boarding home, Frankfurt am Main: Suhrkamp. . (1994). El juego de la viola, Miami, Fla.: Ediciones Universal.

Strausfeld, Michi (2000). "Isla-diáspora-exilio: anotaciones acerca de la publicación y distribución de la narrativa cubana en los años noventa". En Todas las islas la isla: nuevas y novísimas tendencias en la literatura y cultura de Cuba, Madrid: Iberoamericana, 11-23.

Vázquez Montalbán, Manuel (Prolg.) (1996).La Isla contada: el cuento contemporáneo en Cuba, Donostia: Tercera Prensa-HirugarrenPrentsa.

RAE-Diccionario de la Real Academia de la Lengua. (1.09.2015), http://www.rae.es 